\title{
Editorial
}

\section{RADIATION KNOWLEDGE FOR BETTER PATIENT CARE! INTRODUCTION}

Wilhelm Conrad Roentgen, a German physicist, discovered X-ray on November 8, 1895. He was awarded with first Nobel Prize for Physics in 1901.1 At present, the status of the standard of radiology in India is at par with the rest of the world. Routine or specialized interventions, anything you name it, is done here. It has transformed the way how medical illnesses are measured, managed, diagnosed, and treated.

During the last 4 decades, we have seen an evolution of many more sophisticated techniques like ultrasonography (USG), computed tomography (CT), and magnetic resonance imaging (MRI). It has progressed in leaps and bounds and unraveled what was never dreamt before. X-ray departments metamorphosed into imaging departments with quick and accurate access to the inside of the human body. These newer modalities are helpful not only in diagnosis, but also have therapeutic value as well. Advantages of diagnostic tools are saving more lives by facilitating precise and early diagnosis and foretelling the progress of disease. These tools are also helping doctors to achieve best results in treatment and proving an effective tool in the battle against disease.

\section{Difference in these Modalities}

- Radiation is used in X-rays, CT, and mammography-they are invasive and ionizing.

- In USG, sound waves of high frequency are used. They are noninvasive and nonionizing.

- In MRI, magnet is used and proton image of body tissue is seen. It is also noninvasive and nonionizing. On the one side, modern gadgets are increasingly being used; on the other side of this wide horizon of radiations, plain X-rays still remain the backbone in patient care as the initial investigative tool. Many times, diagnosis is not made without the help of an X-ray. Radiation is much more in CT as compared to an X-ray. They belong to a group of radiations called electromagnetic radiations. It is the transport of energy through space as a combination of electric and magnetic fields. ${ }^{2}$

\section{RADIATION SOURCES}

Radiation is useful if properly managed; otherwise, it is harmful if it is used indiscriminately as information about dose and quality is lacking to public and nontechnical staff. Many types of radiations are harmless, but ionizing radiations can severely injure humans. We are exposed to many sources: (1) Natural environmental radiations; (2) man-made-medically employed X-rays constitute the highest source of ionizing radiations. Benefits derived from its application in medicine are indispensable; however, such applications must be made with prudence with regard to reducing unnecessary exposure to patient and personnel.

Other sources of man-made radiation, such as nuclear power generators, nuclear weapons, industrial sources, airport surveillance systems, microwaves, etc., contribute to our annual dose. ${ }^{1-3}$

\section{RADIATION MEASUREMENT}

Radiation can be measured as dose equivalent unit, known as Sievert (Sv). There is background radiation-100 to $120 \mathrm{mSv}$ (milliSievert). Maximum permissible dose of a radiation worker is $5 \mathrm{mSv} /$ year. For a nonradiation worker, it is much less, i.e., $0.5 \mathrm{mSv}$ /year. Lethal dose for gonads is $5 \mathrm{mSv} /$ year, thyroid and skin is $30 \mathrm{mSv} /$ year. Extremities can tolerate a maximum of $75 \mathrm{mSv} /$ year. ${ }^{1,3}$

\section{RADIATION HAZARDS}

As X-rays travel with the speed of light, i.e., 186,000 miles $(3 \times 108 \mathrm{~m} / \mathrm{s})$ and are not seen by human eyes, it causes damage to our body. Radiation damage can occur due to type of exposure: (a) Interrupted, i.e., on and off exposure, or (b) continuous, meaning radiation exposure is continuous. 
Harmful effects of radiation can be of two types: (a) Somatic-damage to the body, and (b) genetic damage that can affect offspring. If the radiation response occurs within minutes or days after radiation exposure, it is classified as an immediate or early effect of radiation. On the contrary, if the human injury is not observable for many months or years, it is termed a delayed or late effect of radiation. High dose in short duration or accumulated dose in long duration can damage the human body. ${ }^{1}$

Damage to body occurs by two methods: (1) Radiolysis of water, (2) damage to nucleic acid.

\section{Radiolysis of Water}

Human body contains approximately $80 \%$ water molecules; irradiation of water represents the principal interaction in the body. When water is irradiated, it dissociates into other molecular products and the action is termed radiolysis of water.

When an atom of water $\left(\mathrm{H}_{2} \mathrm{O}\right)$ is irradiated, it is ionized and dissociates into two ions-an ion pair- $\mathrm{H}^{-}$and $\mathrm{OH}^{-}$, and two free radicals, $\mathrm{H}^{*}$ and $\mathrm{OH}^{*}$. Ions can recombine and therefore no biological damage would occur. A free radical is uncharged molecule containing a single unpaired electron in the valence or outermost shell. Free radicals are unstable and therefore exist with a lifetime of less than a millisecond. The $\mathrm{OH}^{*}$ free radical can join with a similar molecule and form hydrogen peroxide $\left(\mathrm{H}_{2} \mathrm{O}_{2}\right)$ by the following equation: $\mathrm{OH}^{*}+\mathrm{OH}^{*}$ $\rightarrow \mathrm{H}_{2} \mathrm{O}_{2}$. The $\mathrm{H}^{*}$ free radical can interact with molecular oxygen to form hydroperoxyl radical $-\mathrm{H}^{*}+\mathrm{O}_{2} \rightarrow$ $\mathrm{HO}^{*}{ }^{1-3}$

Hydrogen peroxide is a gas and it will damage one cell $\rightarrow$ then many cells $\rightarrow$ many cell means tissue $\rightarrow$ many tissues means an organ $\rightarrow$ now one to many organs $\rightarrow$ if many organs means human body.

Sequence of events following radiation exposure of humans can lead to several radiation responses. At nearly every step, mechanisms for recovery and repair are available. This happens if radiation is continuous; otherwise, there is chance of repair at various levels.

\section{Damage to Nucleic Acid}

There are two principal nucleic acids of importance to human metabolism—deoxyribonucleic acid (DNA) and ribonucleic acid. The DNA serves as command or control molecule for cell function. It contains all the hereditary information representing a cell and, if it is germ cell, then all the hereditary information of the whole individual.

A radiation effect on DNA is the most important in the human body because it contains the genetic information for each cell. Each cell has a nucleus containing DNA complexes with other molecule in the form of chromosomes. The chromosome therefore controls the growth and development of cell, which in turn determines the characteristics of an individual.

A DNA molecule can be damaged without the production of a visible chromosome aberration. It is reversible, and can lead to cell death. If enough cells of the same type respond similarly, then a particular tissue or organ can be destroyed. Damage to DNA can also result in abnormal metabolic activity. Uncontrolled rapid proliferation of cells is the principal characteristic of radiation-induced malignant disease. If damage to DNA in a germ cell occurs, then it is possible that it will not be observed until the following generation or later., ${ }^{1,3}$

Chromosome contains miles of DNA and, therefore, when a visible aberration does appear, it signifies a considerable amount of radiation damage. If radiation damage to the DNA is sufficiently severe, visible chromosome aberrations may be detected-terminal deletion, dicentric formation, and ring formation. In summary, there are three principal observable effects resulting from irradiation of DNA—cell death, malignant disease, and genetic damage.

\section{Human Response to lonizing Radiation}

- Early effects of radiation on humans

- Acute radiation syndrome-hematological, gastrointestinal, and central nervous system (CNS) syndromes

- Local tissue damage-skin, gonads, and extremities

- Hematological depression

- Cytogenetic damage

- Late effects of radiation on humans

- Leukemia 
- Other malignancies-bone, lung, thyroid, and breast

- Local tissue damage—skin, gonads, and eyes

- Life span shortening

- Genetic damage—cytogenetic, doubling, or genetically significant

- Effects of fetal irradiation

- Prenatal death

- Neonatal death

- Congenital malformations

- Childhood malignancy

- Diminished growth and development

\section{QUALITY CONTROL}

There are various national and international radiation regulatory agencies. The International Atomic Energy Agency has surveyed 12 countries and found that $53 \%$ of the X-ray is not useful. Per day, around the world, patients are subjected to more than 10 million diagnosis procedures, 100,000 nuclear medicine procedures, and 10,000 radiotherapy cases. Out of these, $90 \%$ are chest or abdominal X-rays.

Poor-quality images are observed in 4 to $53 \%$ of the images. As far as India is concerned, quality control data show that $10 \%$ were repeated where quality control is practiced and $50 \%$ repeated where quality control is not practiced. It has also shown that, in bedside radiological procedure in a study of 75 countries, about $51 \%$ of the patients were infected.

\section{SAFETY CODE}

All radiological examinations carry a risk, however, small. It is not possible to establish a safe dose threshold below which X-rays are safe. There are laid down safety codes for medical diagnostic X-ray equipment and installations. Widespread utilization of ionizing radiations in multifarious applications-in medicine, industry, agriculture, and research-has brought in its wake the need for exercising regulatory control to ensure safety to the user and to the general public. The Atomic Energy Regulatory Board was constituted in 1983 by the Government of India. Every effort shall be made to reduce the radiation doses to individuals to levels as low as reasonably achievable (ALARA principle). ${ }^{4-6}$

Although no upper limits of radiation exposure are established, every effort must be made to limit each patient's dose. As the physician responsible for ordering examinations, you have the most important role in ensuring the safety of your patient by ordering an examination thoughtfully and responsibly. We offer the following as guidelines which we hope will be helpful to you. Although clinical judgment should always overrule guidelines, in the absence of clear clinical indications to the contrary, guidelines should be followed.

\section{Reducing Patient Dose}

- Criteria for choosing examination: There should be a valid clinical reason for every examination. All $X$-ray studies should be chosen for clinical utility and for no other reason, such as preoperative chest X-ray (CXR) below 45 years of age, mammogram screening below 40 years, and lumbar spine for disk.

- Limit follow-up examination-always consider: How large is the dose, e.g., chest or lumbar spine? How quickly are clinically significant changes likely to occur? Management of pneumothorax often requires frequent but back pain rarely does.

- Be aware of high-dose examinations, like barium enema (radiation is equivalent to one year's dose), fluoroscopy, lumbar spine, CT, intravenous urography. In some, alternative radiological examinations can be ordered. A CT scan with many sequences, contrast, and delayed images gives lot of radiation.

- Limit number of views-responsibility of radiology department after discussion with referring physician.

- Irradiation in pregnancy-as the embryo/fetus is extremely radiosensitive, every effort should be made to reduce fetal dose during a radiology procedure. It has to be decided by a committee of doctors after counseling of husband and wife with written consent. Major risks associated are childhood cancer and CNS abnormalities. 


\section{Other Considerations}

- Concept of detriment by the International Council for Radiation Protection is harm due to radiation exposure, risk of fatal or nonfatal cancer, relative amount of lifetime lost, risk of severe hereditary effects. ${ }^{4-6}$

- Female during first 10 days after cycle. Chest X-ray is equivalent to background radiation dose received in approximately 10 days.

- Contrast material may cause serious and even fatal adverse reactions.

- Relative cost of health care is worldwide concern and it is important for physicians to be aware of the cost of each procedure.

- Interaction with the imaging department: Do not hesitate to contact a radiologist if you need advice. Please complete requisition forms accurately and legibly to help the radiologist to do the best for your patient. We must adopt the policy of constant and regular communication openness with staff and clinicians.

Radiation protection in medical uses, radiation exposure, or treatment should be undertaken only when it offers advantages over other methods.

Conduct workshops, lectures, and teaching programs in hospital. Patients will be immensely benefited by such programs:

- Introduction to radiation

- Hazards and protection or any fear

- Indications

- Views properly asked

- Showing normal and abnormal X-rays

- Patient care department, operating room, emergency room, portable

- Patient preparation

- Difference between other modalities - CT, MRI, or USG

- Improved department hospital atmosphere and relationship

- Less number of X-rays with full clinical information

\section{Role of Physician}

- Short-term courses in diagnostic radiology

- Rapport with specialist and radiologist

- If they are isolated and not interacting with us there is problem in patients diagnosis

- Primary - for X-ray request decision - (a) more clinical, (b) treatment, (c) X-rays - Yes/No? If Yes to continue treatment or refer patient

- Medicolegal problem

- Do not request any X-ray unless clinically justified and likely to affect management of patient

- Proper clinical notes on records

- X-ray requisition by

- Clinical examination, details must precede X-ray

- Provisional or differential diagnosis

— Views needed/discuss

- Repeats and follow-ups

- Serious, irritable, myocardial infarction, traumatic - stabilize patient or do resuscitation first.

- Must know preparation

- Females of reproductive age - last menstrual period 10 days rule to be followed.

Awareness of patient about X-rays is very little. Why? It is because of our fault. Many times patients also insist for X-rays because many think it as curative and majority are not aware of its harmful effects. Unfortunately, it is not the case; indiscriminate use of X-rays to patients has become a day-to-day practice. Mushrooming of X-ray clinics has become a "minting" industry. But who is the sufferer? Naturally, patients only.

Presently we say that if we outweigh the advantages and disadvantages of X-rays, we can conclude that risk is minimal if we do a proper X-ray in ideal conditions. But on the contrary, we say that it is criminal to do any $\mathrm{X}$-ray examination of a patient if clinically not indicated. Any request for X-rays should come after proper evaluation of the case and if it is going to help in clinical diagnosis of the case. 
Overall, in our experience, we found that our interaction with others is very little or not at all. We also found that improper use of X-rays is not uncommon. We feel giving a message to them about radiations is reasonable. Those who do not know about radiation and its hazards will keep on requesting unnecessary $\mathrm{X}$-rays, but when they know it they will restrict them. We emphasized to all of them about usefulness of radiation and if properly used it has negligible side effects.

\section{CONCLUSION}

Any such program has helped in better patient care and better relations with us on a day-to-day basis. We do know that benefits derived from the diagnostic X-rays are enormous. It is our job to produce high-quality images with minimal radiation exposure. This approach can result in the highest benefit and the smallest risk. It is mandatory that the safety stipulations of code are followed by manufacturer, seller, and users of diagnostic X-ray equipment. In any situation, patient's experience with us should be synonymous with transparency, ethics, trust, and empathy. It is a never-ending story; if we keep challenging success, it is forever evolving. Journey starts afresh again so keep exploring angles that others cannot.

\section{REFERENCES}

1. Bushong SC. Radiologic science for technologists - physics, biology and protection. 5th ed. Mosby 1993.

2. Curry TS, Dowdey JE, Murray RC. Christensen's physics of diagnostic radiology. 4th ed. Lea \& Febiger; 1990.

3. Bomford CK, Sherriff SB, Kunkler IH. Walter \& Miler's textbook of radiotherapy, radiation physics, therapy and oncology. 5th ed. Churchill Livingstone 1993.

4. Atomic Energy Regulatory Board. AERB safety code AERB/SC/ MED-2- October 5, 2001 Mumbai.

5. International Atomic Energy Agency. Vienna, 2004.

6. International Council for Radiation Protection. Radiological protection education in medicine: an essential but often missing element. [Ann ICRP 2009.]

Anil K Shukla

Professor

Department of Radiodiagnosis

RajaRajeswari Medical College \& Hospital

Bengaluru, Karnataka, India Phone: +918023442748

E-mail: shookla2007@yahoo.co.in 